\title{
Whispering gallery modes formation in small nanocavities based upon crossed nanobeam structures
}

\author{
Ahmadreza Daraei", Atefeh Mohsenifard, Mohammad Kafi Meibodi \\ Department of Physics, Faculty of Science, University of Sistan and Baluchestan, Zahedan, Iran \\ Email address: \\ Daraei@phys.usb.ac.ir (A. Daraei)
}

\section{To cite this article:}

Ahmadreza Daraei, Atefeh Mohsenifard, Mohammad Kafi Meibodi. Whispering Gallery Modes Formation in Small Nanocavities Based upon Crossed Nanobeam Structures. American Journal of Optics and Photonics. Vol. 2, No. 3, 2014, pp. 28-31.

doi: 10.11648/j.ajop.20140203.12

\begin{abstract}
In this paper, we investigate conditions to form whispering gallery modes (WGMs) in small nanocavities (NCs) which are designed at the intersection area of couple of nanobeams (NBs) structures. Tapered air-holes at each branch of NBs have been employed as a part of mirror accompanied by curved-walls at cross junctions to form a small curved nanocavity. Simulations by finite element based commercial software, COMSOL Multiphysics 4.3 show that confined photonic modes are well established via WGM mechanism. Variation of modes wavelength and their field intensity profiles have been investigated. Quality factor, Q, as high as 198400 is obtained for a particular WGM at mode wavelength $1471.9 \mathrm{~nm}$ with computed modal volume as low as $\mathrm{V}_{\text {mod }} \approx 0.25(\lambda / \mathrm{n})^{3}$.
\end{abstract}

Keywords: Crossed Nanobeam Cavities, Photonic Wire, Whispering Gallery Modes, Curved-Wall Cavity, Tapered Air-Holes

\section{Introduction}

Photonic crystal $(\mathrm{PhC})$ nanocavities $(\mathrm{NCs})$, with variety of geometries, are sort of optical cavities that permit for low modal volumes $\left(\mathrm{V}_{\mathrm{mod}}\right)$ and high quality $(\mathrm{Q})$ factors with extended use in subjects e.g. low-threshold nano-lasers, cavity quantum electrodynamics (QED) and non-linear Optics. For nonlinear optical interactions such as frequency conversion, it is anticipated to have multiple resonant nanostructures with arbitrary frequency separation plus good spatial field overlap [1-4].

The confinement of light to small volumes has essential concerns for the properties of optical emission within the low dimensional region. Photonic bandgap (PBG) materials which are also known as PhCs, offer a medium of achieving strong photon confinement in volumes on the order of $(\lambda / n)^{3}$, where $\lambda$ is the photon wavelength and $n$ is the refractive index of the host material [1-2,4-6].

Optical components such as ultrahigh-Q cavities and high reflectivity mirrors have formed the basis of many optical devices including lasers and other types of oscillators [2,3]. In the past decade, vast efforts have been made to employ the current semiconductor technology in micro- and nano- fabrication to more reduce the size of optical and photonic components towards photonic integrated on-chip devices [6-7]. The high-Q PhC NCs with high reflectivity micron-scale mirrors in different low dimensional structures are examples of this technology. In addition, there are other miniaturized technologies with balances in quality of confinement compared to size of resonator, including micropillars, microdisks, etc [8-14]. These technologies can help to confine light in all three dimensions within particular modes through whispering gallery or Fabry-Pérot [11-14].

Whispering gallery modes (WGMs) are the electromagnetic waves which undergo total internal reflection (TIR) in the rounded dielectric materials and retain ultra-high $\mathrm{Q}$ factor. The WGMs have attracted much attention in the area of photonic integrated circuits and optical logic devices [11-14].

A narrow rectangular cross section waveguide with one array of periodic air-holes along its axis is known as photonic wire or nanobeam (NB). Design, fabrication, and experimental characterization of $\mathrm{PhC}$ nanobeams (NBs) containing cavities for applications from optical interconnect to biochemical sensors in variety of structures were goals of extensive researches [15-18].

In this paper, we discuss $\mathrm{PhC}$ design in nanotechnologies, which use tapered distributed Bragg reflectors (DBRs) for confinement in one dimension, and TIR in two other 
dimensions. It is also possible to have NCs included in the middle of the structure, for example by modifying central part of a the narrow photonic wire or NBs with sets of perfectly periodic or tapered air-holes[4,6,7,18-20]. Also, in this paper, conditions for formation of whispering gallery modes in the small nanocavities are investigated. Structures are designed in a way that at the crossed region of two modified nanobeams, confined WGMs to be examined. Finite element based commercial software, COMSOL Multiphysics version 4.3, has been used for simulations [21]. Variation of modes wavelength and their field intensity profiles have been explored as well.

\section{Nanobeam Structure and Design}

Structures of NBs which used in this paper include photonic wires in the silicon material. Fig. 1(a) shows a typical schematic of orthogonal NBs (crossbeam cavity) with a central cavity design at intersection region of orthogonal photonic wire including tapered air-holes mirrors at each branch of the NBs. This kind of structure, in principle, may support orthogonal modes with high spatial overlap for frequency conversion in nonlinear optics [4,15-17]. A typical mesh for our simulations is also shown in the Fig. 1(b).

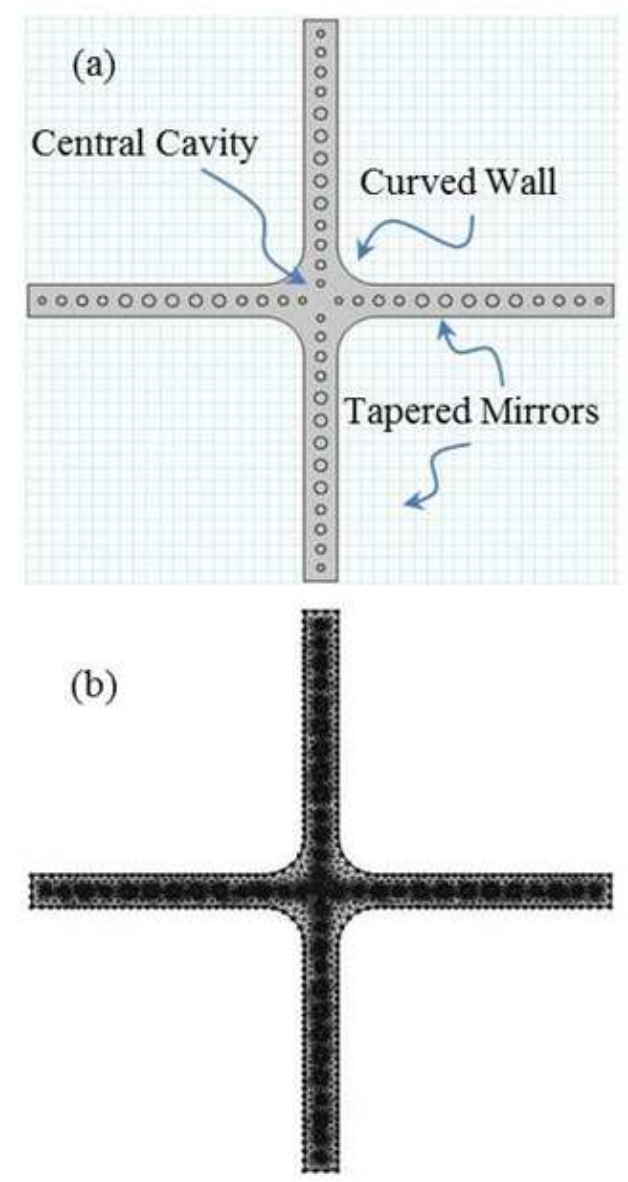

Figure 1. a) Schematic of a central cavity design at intersection region of orthogonal photonic wire including tapered air-holes DBRs at each branch of the NBs, b) A typical mesh for our crossed NB simulations
A curved cavity is formed in the central region by introducing quarter circles at the junctions, with sharing some tapered air-holes of NBs in the NC region in the arrangements. The lattice constant and air-hole sizes near to and far from cavity region are tapered. In each branch confinement along the periodic direction is provided by distributed Bragg reflection by the tapered DBRs and confinement out of plane is provided by TIR. Confinement in the in-plane direction orthogonal to the beam axis is also provided by TIR and in the case of beams with overlapping PBGs, also by distributed Bragg reflection for minimizing crosstalk in waveguide intersections [4].

\section{Results and Discussions}

In this paper, the WGMs in PhC crossed NBs cavities are simulated and analyzed which provided high-Q factors and could be integrated with the other photonic devices. High-Q factors are achieved from these modes by suppressing both the in-plane and the out-of-plane optical losses. The WGMs discussed here are transverse electric (TE) modes which are supported due to choosing proper dimensions and PBG structures. The structure has air-holes periodicity along NBs directions which can produce 1D TE PBG with size determined by the difference in frequency between the first two bands (dielectric and air band).

It should be noted that the PhCs can have multiple PBGs, but the size of the higher order band gaps greatly diminishes for finite thickness structures. Furthermore, in such structures, higher order band gaps are located above the light line, implying that the resonances at those frequencies would also have low-Q factors, resulting from the lack of TIR confinement. Band gaps for different polarizations (e.g. TE and TM) can generate additional resonant modes. However, it is difficult to independently tune their frequencies, and TM resonances require relatively thick membranes that are more difficult to fabricate. A cavity with orthogonally polarized resonances degenerate in frequency can also be formed by using the same parameters for each NB.

As we can see from the $2 \mathrm{D}$ electric field $\left(E_{z}\right)$ patterns obtained by FEM simulations and shown in the Fig. 2(a), a WGM is supported in a typical $\mathrm{PhC}$ crossed NBs cavity structure. In simulations, to optimize the design, parameters of curvature of wall (R), NB cavity length (l), air-holes lattice constant (a), number of periods in tapers inside and outside $\left(\mathrm{N}_{\mathrm{TI}}\right.$ and $\mathrm{N}_{\mathrm{TO}}$ respectively), distance between air-holes in the tapers $\left(\mathrm{a}_{\mathrm{i}}, \mathrm{i}=1,2, \ldots 4\right)$, air-hole diameters in the taper region $\left(\mathrm{d}_{\mathrm{i}}, \mathrm{i}=1,2, \ldots, 4\right)$, and NB width (w) were varied in each NB.

Fig. 2(b) shows the 3D mode map of electric field $\left(E_{z}\right)$ for the same mode at wavelength $1522.9 \mathrm{~nm}$. The simulations indicate that these structures possess computational modal volume values as low as $\mathrm{V}_{\text {mod }} \approx 0.27(\lambda / \mathrm{n}) 3$ (where $\mathrm{n}$ is the refractive index) and cavity $\mathrm{Q}$ factors is as high as $\mathrm{Q} \approx 196860$ for the WGM with that wavelength. 
As we can see in the Fig. 2(c), (e) and (g), the $\mathrm{S}_{\mathrm{x}}$ and $\mathrm{S}_{\mathrm{y}}$ components of the second-, third- and fourth- order TE-like modes supported by the crossed NB are shown respectively. The low Q value in some modes is attributed to scattering losses arising from the mode profile mismatch between the localized cavity mode and the evanescent Bloch mode inside the DBRs. The middle column mode maps, $\mathrm{S}_{\mathrm{y}}$, show degenerate frequency compared with $\mathrm{S}_{\mathrm{x}}$. The 3D mode maps relevant to the first column $\left(\mathrm{S}_{\mathrm{x}}\right)$ for more clarity are shown in d), f) and $h$ ).
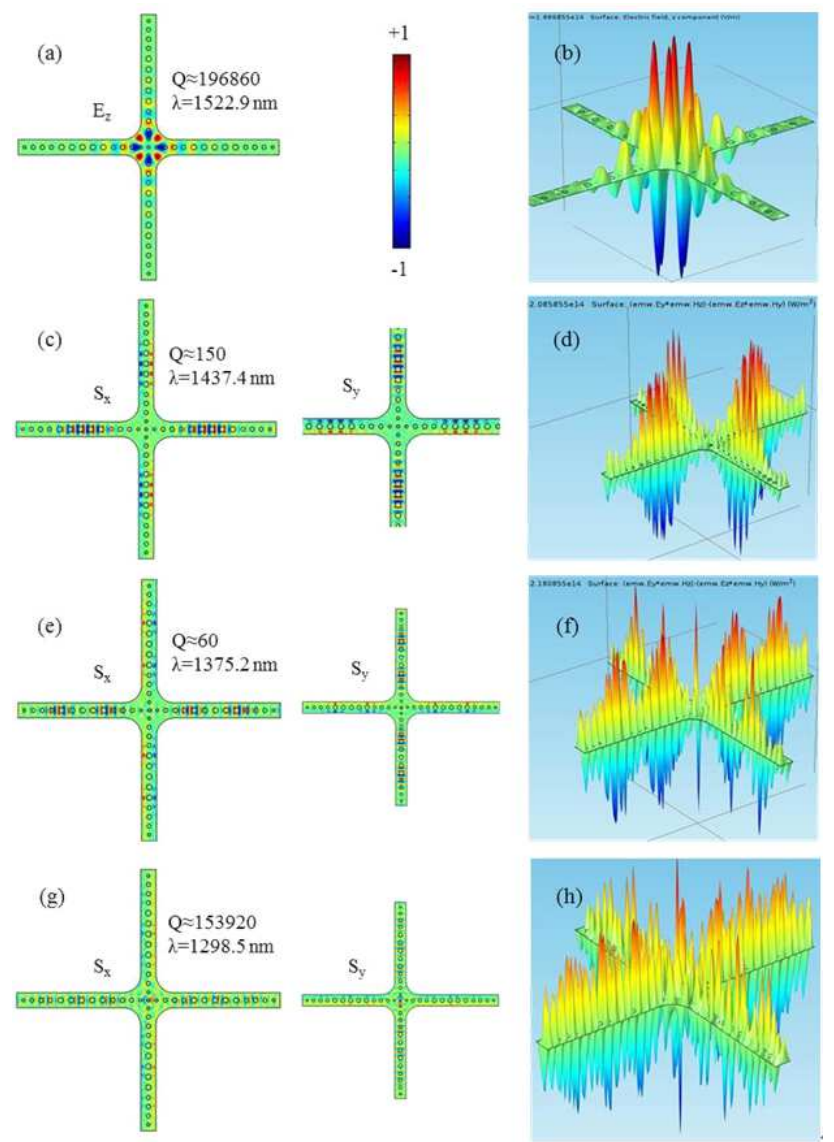

Figure 2. a) 2D, b) 3D, mode maps for electric field ( $\left.E_{z}\right)$ of the fundamental mode (circles indicate air-holes). The $S_{x}$ and $S_{y}$ components of the Poynting vector for c) second-e) third-and $g$ ) fourth-order TE-like modes supported by the crossed $N B$ with parameters: $d_{1, T I}=101, d_{2, T 1}=136$, $d_{3, T I}=150, \quad d_{4, T I}=140 \mathrm{~nm}, \quad a_{1, T I}=290, \quad a_{2, T I}=310, \quad a_{3, T I}=304, \quad a_{4, T I}=342$, $d_{1, T O}=140, d_{2, T O}=150, \quad d_{3, T O}=136, \quad d_{4, T O}=101, \quad a_{1, T O}=342, \quad a_{2, T O}=304$, $a_{3, T O}=310, a_{4, T O}=290, d_{N}=182 \mathrm{~nm}, a_{N}=350 \mathrm{~nm}$, refractive index $n=3.19$, $N=5$; radii of the curved-walls are $600 \mathrm{~nm}$. The middle column show degenerate frequency by $S_{y}$ compared with $S_{x}$ d), f) and h) 3D mode maps relevant to the first column for more clarity

A computational spectrum including marked fundamental-, second-, third- and fourth- order TE-like WGMs supported by the crossed NBs which were discussed in the Fig. 2(a)-(h), is shown in the Fig. 3. Higher wavelength modes are also shown in the figure as well. Some of the NB cavity WGM demonstrated here present simultaneous ultrahigh $Q$ factor and very small modal volume.

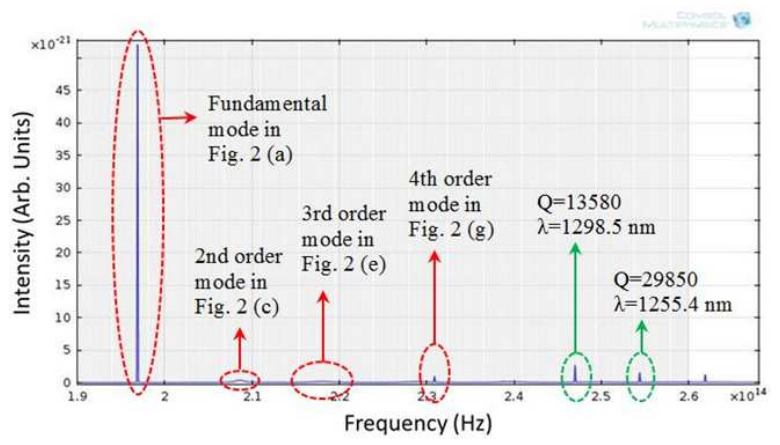

Figure 3. Fundamental-, second-, third-and fourth-order TE-like WGMs supported by the crossed NBs which were discussed in the Fig. 2 (a)-(h), are marked.

To have more insight into the varieties of modes in the crossed NBs, 2D profiles of electromagnetic fields and Poynting vector are examined by varying parameters, e.g. radii of the curved-walls. The $\mathrm{V}_{\bmod } \approx 0.25(\lambda / \mathrm{n})^{3}$ and $\mathrm{Q} \approx 198400$ are achieved for a WGM at $\lambda=1471.9 \mathrm{~nm}$, choosing $700 \mathrm{~nm}$ for radii of the curved-walls, and the results are shown in the Fig. 4.
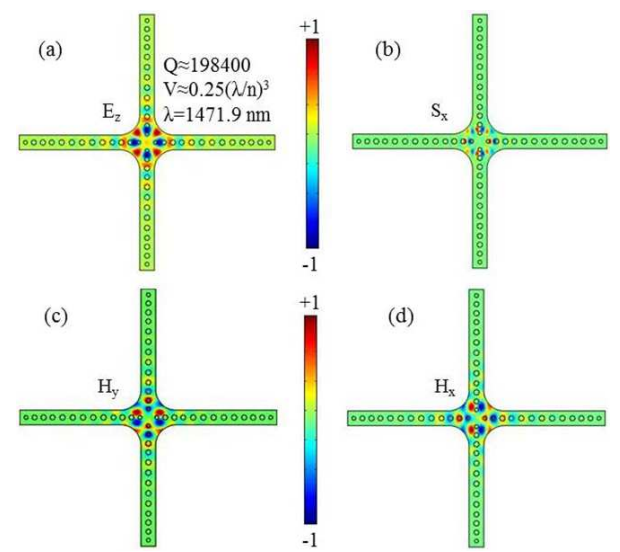

Figure 4. Formation of the WGM in the crossed $N B S$, with $N_{T O}=4$ and $N_{T I}=4.2 D$ profile of component of a) electric field, $\left.E_{z}, b\right)$ Poynting vector, $S_{x}$, and magnetic field c) $H_{y}$, and d) $H_{x}$, of the resonant mode while radii of the curved-walls are $700 \mathrm{~nm}$

\section{Conclusions}

In this paper, we presented circumstances to have WGMs in variety of small NCs which are designed at the intersection area of couple of nanobeams (NBs) structures. At each branch of NBs, mixed tapered air-holes have been employed as a part of mirror accompanied by quarters of circle-shape curved-walls at cross junctions to form a small curved nanocavity. Simulations are done using finite element based software, COMSOL Multiphysics 4.3, and indicated that confined photonic modes are well established via WGM formation mechanism. Variation of modes wavelength and their electromagnetic field intensity profiles over the structures have been investigated. Maximum $\mathrm{Q}$ value and minimum modal volume, for a particular WGM at mode wavelength $1471.9 \mathrm{~nm}$ were 198400 and $0.25(\lambda / \mathrm{n})^{3}$ respectively. 


\section{References}

[1] B. E. A. Saleh, M. C. Teich, "Fundamentals of Photonics", John Wiley \& Sons, Inc., New Jersey, USA, 2nd ed., 2007.

[2] J. D. Joannopoulos, S. G. Johnson, J. N. Winn, R. D. Meade, "Photonic Crystals: Molding the Flow of Light", Princeton University Press, New Jersey, USA, 2nd ed., 2008.

[3] A. Yariv, P. Yeh, "Photonics - Optical Electronics in Modern Communications", Oxford University Press, Inc., New York, USA, 6th ed., 2007.

[4] K. Rivoire, S. Buckley, and J. Vučković, "Multiply resonant photonic crystal nanocavities for nonlinear frequency conversion", Opt. Express, Vol. 19, Issue 22, pp. 22198-22207, 2011.

[5] Y. Zhang and M. Lončar, "Submicrometer diameter micropillar cavities with high quality factor and ultrasmall mode volume", Opt. Letters 34, pp. 902-904, 2009.

[6] M. Radulaski, T. M. Babinec, S. Buckley, A. Rundquist, J. Provine, K. Alassaad, G. Ferro, and J. Vučković, "Photonic crystal cavities in cubic (3C) polytype silicon carbide films", Opt. Express, Vol. 21, Issue 26, pp. 32623-32629, 2013.

[7] A. R. Md Zain, N. P. Johnson, M. Sorel and R. M. De La Rue, "Ultra high quality factor one dimensional photonic crystal/photonic wire micro-cavities in silicon-on-insulator (SOI)", Opt. Express, Vol. 16, Issue 16, pp. 12084-12089, 2008.

[8] A. Daraei et al., "Control of polarization and mode mapping of small volume high Q micropillars", J. Appl. Phys. 102, 043105, 2007.

[9] A. B. Young, R. Oulton, C. Y. Hu, A. C. T. Thijssen, C. Schneider, S. Reitzenstein, M. Kamp, S. Höfling, L. Worschech, A. Forchel, and J. G. Rarity, "Quantum-dot-induced phase shift in a pillar microcavity", Phys. Rev. A, 84, 011803(R), 2011.

[10] M. Schwab, H. Kurtze, T. Auer, T. Berstermann, M. Bayer, J. Wiersig, N. Baer, C. Gies, F. Jahnke, J. P. Reithmaier, A. Forchel, M. Benyoucef and P. Michler, "Radiative emission dynamics of quantum dots in a single cavity micropillar", Phys. Rev. B, 74, 045323, 2006.
[11] M. Bürger, M. Ruth, S. Declair, J. Förstner, C. Meier, and D. J As, "Whispering gallery modes in zinc-blende AlN microdisks containing non-polar GaN quantum dots", Appl. Phys. Lett. 102, 081105, 2013.

[12] E. Peter, I. Sagnes, G. Guirleo, S. Varoutsis, J. Bloch, A Lemaître, and P. Senellart, "High-Q whispering-gallery modes in GaAs/AlOx microdisks", Appl. Phys. Lett. 86, 021103, 2005.

[13] Po-T. Lee, Tsan-W. Lu, Chia-M. Yu, and Chung-C. Tseng, "Photonic crystal circular-shaped microcavity and its uniform cavity-waveguide coupling property due to presence of whispering gallery mode", Opt. Express, Vol. 15, Issue 15, pp. 9450- 9457, 2007.

[14] Han-Y. Ryu and M. Notomi, "High quality-factor whispering-gallery mode in the photonic crystal hexagonal disk cavity", Opt. Express, Vol. 12, Issue 8, pp. 1708-1719, 2004.

[15] F. Liang, N. Clarke, P. Patel, M. Lončar, and Q. Quan, "Scalable photonic crystal chips for high sensitivity protein detection", Opt. Express, Vol. 21, No. 26, pp. 32306-32312, 2013.

[16] Q. Quan, P. B. Deotare, and M. Lončar, "Photonic crystal nanobeam cavity strongly coupled to the feeding waveguide", Appl. Phys. Lett. 96, 203102, 2010.

[17] Y. Halioua, A. Bazin, P. Monnier, T. J. Karle, G. Roelkens, I. Sagnes, R. Raj, and F. Raineri, "Hybrid III-V semiconductor/silicon nanolaser", Opt. Express, Vol. 19, No. 10, pp. 9221-9231, 2011.

[18] P. B. Deotare, M. W. McCutcheon, I. W. Frank, Khan, M. Lončar, "High Quality Factor Photonic Crystal Nanobeam Cavities", Appl. Phys. Lett. 94, 121106, 2009.

[19] S. Kim, B-H. Ahn, J-Y. Kim, K-Y. Jeong, K. S. Kim, and Y-L. Lee, "Nanobeam Photonic Bandedge Lasers", Opt. Express, Vol. 19, Issue 24, pp. 24055-24060, 2011.

[20] K. Rivoire, S. Buckley, and J. Vučković, "Multiply resonant high quality photonic crystal nanocavities", Appl. Phys. Lett. 99, 013114, 2011.

[21] COMSOL Multiphysics 4.3, http://www.comsol.com/. 